\title{
"Patria, cultura, justicia y libertad": Alex Curling Delisser en los albores de la intelec- tualidad negra y caribeña en Costa Rica
}

\author{
"Homeland, Culture, Justice and Freedom": Alex Curling \\ DELISSER AT THE DAWN OF THE BLACK CARIBBEAN INTELLIGENTSIA \\ IN COSTA RICA
}

Marianela Muñoz Muñoz

Universidad de Costa Rica, San José, Costa Rica marianela.munoz@ucr.ac.cr

Diana Senior Angulo

Universidad de Costa Rica, San José, Costa Rica diana.senior@ucr.ac.cr

Resumen: El artículo analiza la trayectoria y pensamiento de Alex Curling Delisser desde una identidad negra y caribeña en Costa Rica. Como intelectual y primer diputado descendiente de inmigrantes jamaicanos, Curling enfrentó a una matriz cultural en la que el ejercicio del poder, cifrado en términos de "raza" (etnicidad), concedía a la vez que estructuraba a priori un

1 Algunos fragmentos de este artículo son una adaptación de lo expuesto en la tesis doctoral Bilingüismo politico: Afrocaribeñas en el estado blanco y multicultural costarricense (1978-2017).

2 La escritura colaborativa de este artículo se facilita en el marco del Programa "Connected Worlds: The Caribbean, Origin of Modern World", financiado por el European Union's Horizon 2020 programme for research and innovation (Marie Sklodowska Curie grant agreement No 823846). 
lugar socialmente diferenciado para esta población. El empleo del recurso de la prensa, siguiendo un modelo de circulación de ideas de tradición circum-caribeña, junto al contenido de su discurso político, sugieren una permanente concientización, dirimida en términos colectivos y políticos, en defensa de la ciudadanía para los "costarricenses de color". Igualmente, su trabajo da cuenta de una erudición y manejo de referentes políticos negros y de otras narrativas culturales locales y de carácter "cosmopolita". Pese a un reconocimiento de sus contribuciones como "padre de la igualdad jurídica” en el país, el carácter diaspórico del discurso de Alex Curling, la negociación de su identidad "nacional" y su relación con una intelligentsia caribeña no han sido debidamente estudiados. El análisis de algunas de sus intervenciones escritas pretende activar esta discusión.

Palabras clave: intelectualidad, identidad negra, prensa, ciudadanía, Caribe costarricense, pensamiento político negro.

AвstRACt: This article analyzes the trajectory and political thought of Alex Curling Delisser from a Black, Caribbean identity in Costa Rica. As an intellectual and the first West Indian descendent congressmen, Curling faces a racialized matrix of power where his community is previously defined and socially differentiated. Curling's use of the press, following a circum-Caribbean tradition of circulation of ideas, in tandem with the content of his political discourse, suggests a permanent awareness and defense of Costa Rican citizenship for people of color. Complementary, his work shows an erudition and active use of Black political references, next to other local and "cosmopolitan" cultural narratives. Despite the fact that Alex Curling has been recognized as the "Father of legal equality" in Costa Rica, the diasporic nature of his speech, the negotiation of his "national" identity and his relationship with a Caribbean intelligentsia have not been dually studied. The analysis of some of his written interventions aims to activate this discussion.

Keywords: intellectuality, black-identity, press, citizenship, Costa Rican Caribbean, Black political thought. 


\section{INTRODUCCIÓN}

En el año 2000, por iniciativa de la diputada Joycelyn Sawyers Royal, se declara al Lic. Alex Curling Delisser como Benemérito de la Patria y "Padre de la igualdad jurídica" en Costa Rica. Se trata de un esfuerzo de la comunidad afrocaribeña ${ }^{3}$ por honrar su memoria y contribución. En palabras de la proponente del proyecto, tal fue uno de sus propósitos en su llegada al Congreso: "No me voy de aquí, sin dejar un padre de la patria negro". Su preocupación: "Ese hombre estaba casi olvidado [...] y no solo el negro, fueron las minorías, las minorías de este país. Es un gran hombre y costó sacarlo" (Sawyers, "Comunicación personal”) ${ }^{4}$. Para Duncan y Allen:

Don Alex Curling combatió reciamente contra el Art. 5to Ley 31 de 1934, que restringió la movilidad del negro hacia el Pacífico con motivo del traslado de la compañía; dictó charlas en el seno del Centro para el Estudio para los Problemas Nacionales sobre el tema del negro la necesidad de un trato igual para todos. Después de que la Junta de Gobierno abolió las disposiciones discriminatorias, siguió defendiendo con cartas al Presidente Ulate (La Nación, 20.8.1950:4) y ante otros órganos del Estado (La Nación, 20.11.1949:4) hasta que al final él mismo en su condición de diputado suplente electo al Congreso, promovió una ley sobre naturalizaciones que lo ha hecho merecer el título de Padre de la Igualdad Jurídica en Costa Rica (Duncan y Allen 35).

3 En este artículo, se utilizarán los términos negro y afrocaribeño para aludir a la matriz etno-identitaria previa a la reconfiguración afrocostarricense, lograda por medio de la ciudadanía. La comunidad de descendientes de migrantes antillanos -principal, aunque no exclusivamente jamaicanos- en Costa Rica se autoidentifica, hasta el día de hoy, como afrocostarricense, afrocaribeña y negra, de manera intercambiable. Ahora bien, en el contexto de emergencia del discurso de Alex Curling, él mismo emplea la expresión "gente de color", por lo cual se incluye también este apelativo en la exposición argumentativa.

4 Además de conseguir el reconocimiento de Alex Curling como primer Benemérito de la patria afrocostarricense y Padre de la igualdad jurídica, la diputada afrocostarricense Joycelyn Sawyers fue la proponente de la Reforma del Artículo 1 para reconocer el carácter pluricultural y multiétnico de la nación. El análisis de su discurso y el de otras diputadas representantes de esta comunidad representa un espacio fértil para la identificación de un pensamiento político afrocostarricense. 
Curling Delisser es descendiente e integrante de una comunidad migrante, negra y caribeña en Costa Rica, zanjada en términos de "raza" (etnicidad) y clase. Dicho contingente, así como sus descendientes, compartieron y dinamizaron la identidad nacional de la "gente de color", a nivel local. Al mismo tiempo, participaron de un contexto intelectual internacional de carácter circum-caribeño, el cual termina por avivarse en Costa Rica a partir de reivindicaciones alrededor de los derechos y el acceso a la ciudadanía.

Si bien las contribuciones de Curling en la esfera política han sido reciente y tímidamente recuperadas, su pensamiento e ideas en la prensa periódica no han sido analizadas en conjunto hasta el momento. De ahí que, mediante la revisión de las publicaciones firmadas a título personal, este artículo examina las contribuciones de Alex Curling en términos de una intelectualidad étnica. Se explora el influjo de la tradición circum-caribeña en su escritura política en la prensa, la circulación de ideas políticas y culturales de la diáspora, los referentes del activismo y cultura negra, junto a otras figuras y conocimientos de carácter internacional o "cosmopolita". Y, de manera complementaria, las dinámicas de concientización que, a todo nivel, promueven la afirmación y el derecho a una ciudadanía negra y costarricense.

La investigación del pensamiento político de Curling pretende dar cuenta de los desafíos de una comunidad que reclamaba su condición ciudadana, mientras su "raza" (etnicidad) y sus marcas culturales (como la anglofiliación) colisionaban con el arquetipo "blanco" e hispánico como epítome de la democracia costarricense.

El análisis resalta la aparición, agenda y discurso de Curling no como acción individual, sino relacionada con la historia cultural y política de la comunidad de migrantes caribeños y sus mismos vínculos con otras comunidades, organizaciones y luchas negras a través de la diáspora. Adicionalmente, la argumentación reconoce los mismos imaginarios de la democracia blanca, patriarcal, igualitaria y excepcional costarricense ${ }^{5}$

Los discursos fundacionales de la identidad costarricense abrazan una narrativa de homogeneidad racial blanca. Sobre la génesis del nacionalismo blanco en Costa Rica, ver los trabajos de Palmer, "Hacia la 'auto-inmigración'. El nacionalismo oficial en Costa Rica 1870-1930"; de Quesada Soto, Uno y los otros. Identidady literatura en Costa Rica 1890-1940; de Molina Jiménez, Costarricense por dicha: identidad nacional y cambio cultural en Costa Rica durante los Siglos XIX y XX; y 
como el telón de fondo de los complejos y dispares escenarios políticos, individuales y colectivos, de la población actualmente reconocida como afrocostarricense.

CURLING COMO HIJO E INTELECTUAL DE SU CONTEXTOY COMUNIDADÉTNICA

Acercarse a la figura de Alex Curling Delisser exige la consideración de los desafíos que, tanto en el plano de las identidades como en el de la incorporación social, económica y política, enfrentó su comunidad de origen en las naciones receptoras de la época. Esto es, los procesos de llegada, permanencia y negociación de ciudadanía de la población migrante del Caribe a lo largo de los litorales e incluso capitales centroamericanas durante la primera mitad del siglo XX.

En un primer plano, resulta entonces relevante la propia inscripción de Curling en una colectividad diaspórica, la cual, en tanto caribeña, sugiere un proceso de conciencia grupal, un sentido de pertenencia y de solidaridad (Isajiw 35; Premdas 10). Como parte de una comunidad de descendientes del Caribe negro, Curling asume y dinamiza una identidad étnica (González 295) ${ }^{6}$, la cual, desde su carácter de trans-locación, relativiza la esfera de lo nacional para el accionar político (Muñoz Muñoz, "Afrocentroamericaneidades" 54). Este grupo comparte un repertorio de hechos históricos, tales como la esclavitud, las plantaciones y el colonialismo (Premdas 11). Más aún, la relectura y cuestionamiento de tales experiencias de violencia y racialización y la actualización de sus manifestaciones, motivan una movilización política negra circum-caribeña en el marco del llamado periodo entreguerras (Putnam, Radical 3); precisamente, durante la antesala de la formación intelectual y política de Alex Curling Delisser.

de Putnam "Ideología racial, práctica social y Estado Liberal en Costa Rica". Sobre la relación del Caribe limonense y la identidad negra, ver Hutchinson Miller, The Province and Port of Limon: Metaphors for Afro-Costa Rican Black Identity.

6 Para González, la "identidad étnica” se define "como un tipo de representación colectiva que se distingue de otras por el manejo simbólico de la diferencia grupal con el fin de defender intereses concretos". Constituye, además, "una consecuencia del trabajo mental de los intelectuales étnicos” (González 294-295). 
De manera particular y en virtud de una escalada en las manifestaciones racistas de carácter supranacional, hombres y mujeres de color propugnan el deber de elevarse desde distintos frentes de organización comunitaria y diaspórica. Ubicados principalmente en el Caribe costarricense, participan en los circuitos del internacionalismo negro, los cuales entrelazan una comunidad imaginaria transnacional no hispánica a lo largo de las naciones "bananeras". En palabras de Putnam, "places like Colón, Limón, and Santiago de Cuba were not only located within Spanish speaking republics: they were located within a supranational black public sphere, within which Afro-North Americans and Afro-Caribbeans spoke to each other literally and figuratively" (Radical 194). Dicha dinámica también incluyó discusiones supranacionales sobre los destinos colectivos, vía prensa y movilizaciones panafricanistas como el garveyismo, principalmente, luego de la depresión de 1929. Así, tanto la UNIA ${ }^{7}$ como la prensa funcionaron como plataformas culturales que nutrieron el pensamiento y condujeron el accionar político de generaciones negras caribeñas y de sus descendientes.

En un segundo plano, Curling y su comunidad de origen enfrentan el dilema de regresar a sus islas de origen, emigrar hacia otro destino o adoptar la ciudadanía de la nación receptora. Se trata de un proceso paulatino que inicia a mediados de la década de los treinta y durante los cuarenta en donde coinciden, por un lado, fenómenos como el fin del boom bananero, la crisis económica y el aumento de restricciones migratorias para los descendientes y pobladores (negros) de las colonias de un Imperio en decadencia (Putnam, Radical 120-121). Por otro lado, y a nivel local, se vive una agitación a causa de los sucesos anteriores y posteriores a la Guerra Civil del 48 en Costa $\mathrm{Rica}^{8}$. La opción por la

7 Resulta clave el papel de la Universal Negro Improvement Association (UNIA) en la articulación de la comunidad. Pese a la diversidad del contingente negro costarricense y en virtud de una urgencia de los destinos de la "raza" de color en suelo costarricense y más allá de él, hacia fines de la década de los veinte, la UNIA se convierte en el dinamizador de un orgullo racial (Chomsky 202; Senior 29).

8 En el contexto de la última guerra civil costarricense en 1948 y el inicio de la llamada Segunda República, el líder del Partido de Liberación Nacional, José Figueres Ferrer, respondió a las demandas de los inmigrantes afrocaribeños y demás comunidades negras de la región "Atlántica” para asegurar su victoria en las elecciones presidenciales. Mediante el pacto establecido con Figueres y su proyecto de "nacionalización" de la provincia de Limón, se inaugura una histórica relación entre el bloque de mayor poder en la historia nacional, el Partido 
ciudadanía costarricense, si bien no la única, representó una de las rutas más accesibles y respaldadas por una comunidad negra que se movilizó para asegurar sus procesos de naturalización y nacionalización. Senior analiza cómo el proceso de adquisición de la ciudadanía por parte de la llamada población afrocaribeña a través de la naturalización durante el periodo 1927 a 1963 se acompaña, además, de un proceso de "nacionalización" que conlleva la identificación de y con las narrativas oficiales y la consecuente, aunque limitada, incorporación sociocultural y política al país (243-244).

Desde ambas coordenadas, una herencia identitaria étnica negra-caribeña y una negociación de ciudadanía negra-costarricense, es posible enmarcar la emergencia de una figura como Curling Delisser. Particularmente, sirven para comprender su pensamiento político e, inclusive, su participación en una especie "intelligentsia étnica" caribeña, la cual se entiende dentro de un contexto analítico mayor como "grupo étnico corporado" y/u organizado (González 274). Este grupo muestra "comportamientos etnicistas", los cuales son "producto tanto de la epistemología en la que fundan su comportamiento, como por los grados y tipos de instrucción adquirida" (González 293). En el caso de la comunidad de descendientes antillanos, estas prácticas incluyen el manejo de los discursos de respetabilidad y orgullo negro, los cuales serán fundamentales para demostrar su condición "honorable", digna y trabajadora. Apelar a este comportamiento étnico y sus narrativas confronta una escalada de manifestaciones antinegras en Costa Rica, las cuales incluyen reclamos y descalificaciones de los costarricenses que consideraron la presencia de los migrantes jamaicanos en el espacio laboral como afrenta de sus derechos como trabajadores blancos costarricenses; señalamientos de líderes políticos y de base quienes asociaron la anglofiliación de la comunidad con la expansión del imperialismo estadounidense, o bien, de intelectuales que manifestaron su preocupación por el "ennegrecimiento" de la sangre costarricense, bajo la influencia del racismo científico y teorías sobre la eugenesia. Uno de los episodios cruciales que provocaron la reacción de la misma intelligentsia

Liberación Nacional y la comunidad afrocostarricense (Duncan y Allen 81-82).

9 En intercambio epistolar entre el Dr. Clodomiro Picado Wight y el escritor Ricardo Fernández Guardia (ambos intelectuales reconocidos por la historiografía costarricense), el primero le comparte al segundo su preocupación por que " ¡la sangre costarricense se está oscureciendo!" (Senior 73). 
y comunidad étnica ocurre ante el traslado de las operaciones de la United Fruit Company al Pacífico, hacia mediados de la década de los treinta. Mientras los trabajadores "blancos" de las bananeras protestaron como "auténticos costarricenses" ante la posibilidad de "que los negros invadieran la zona bananera del Pacífico", más de cien afrocaribeños firman una petición dirigida al Congreso reclamando el despojo de sus derechos como ciudadanos ${ }^{10}$.

La identidad étnica funciona así distinguiéndose de otras identidades "por constituir una representación colectiva sobre la base de prácticas intelectuales, es decir, como resultado de un comportamiento ligado a la alta instrucción" (González 294). Como ejemplo de estas estrategias de movilización de corte intelectual y "estando convencidos de la gran necesidad para la adecuada diseminación de actividades culturales entre sus similares raciales", Miss Verónica Spoyser le sugiere "a un número de miembros de la colonia afrocaribeña anglófona residente en San José", la inauguración de una organización que funcionara bajo la designación o título de: "Centro Cultural Afro-costarricense". Quienes decidieron asociarse a este "valioso movimiento" fueron: las Misses Elena Clarke, Hazel Hudson, Hielen Hudson, Albertha Dobson, Doris Davis, Louise Kindness, Berencia Chen, Evelyn Mc Donald, Elena Douglas, Ethel Gillings, Ruth Rodríguez, Vera Johnson, Gwendolyn Fennell, Amy Aird, Edna Louis, Marie Louise Lyew, Flora Curling (hermana de Alex Curling), Mrs. Evadne Brown, Messrs. Albert Lewis, Rupert Brown, George Mc Donald, Jacob Petgrave, Roland Pugh, Lewis Benson, Sydney Taylor, George Curling (hermano de Alex Curling), Osborne McKenzie, Ferdinand Todd, Thomas Allen, Cedric Kerr, Alex Curling, Benjamín Stanley, Esther Henry, Garfield Walker, Joseph Grant, Henry Booth y Frank Drummond. Dentro del primer personal administrativo del Centro, electos para servir por un término de seis meses estuvieron: Mr. Thomas Allen, presidente; Mr. Rupert Brown, vicepresidente; Mr. Alex Curling, secretario; Miss Verónica Spoyser, dama presidenta, y Miss Elena Clarke, dama secretaria ${ }^{11}$ (Senior 127-128).

10 La carta se envía a los diputados representantes de la provincia limonense el 1 de diciembre de 1934; pero nueve días después se aprueba la Ley $\mathrm{N}^{\circ} 10$ de Contratación Bananera de 1934, la cual en su artículo quinto prohíbe la contratación de mano de obra negra en el Pacífico (Senior 66).

11 El archivo sobre la creación del "Centro Cultural Afrocostarricense" puede encontrarse en La Voz del Atlántico, [Limón, Costa Rica], 16 de mayo. 1942: 6. 
Tomando en cuenta la propuesta de Said en relación con la figura del intelectual crítico y comprometido y su papel más allá de su profesión o responsabilidad como individuo (Said 19-20), llama la atención el involucramiento de Curling y su familia en este grupo. Su protagonismo en medio de debates y movilizaciones de su comunidad irá perfilando, también, su liderazgo político; ello, tanto en relación con los procesos de ciudadanía, como de participación en los espacios de toma de decisiones. Gracias a esa incidencia antes, durante y después de su llegada al Congreso, podemos identificar el peso de la conciencia étnica en su discurso publicado en prensa o pronunciado ante el plenario de la Asamblea Legislativa. Pero, además, junto a tales recursos, como seńala González, es posible identificar otras ideas y comportamientos "cosmopolitas, propios de la intelectualidad" (293). Estas otras referencias y sus aparentes tensiones con las mismas "conductas étnicas" se comprenden en la medida que el intelectual "negro" debe operar y negociar con los imaginarios de la democracia blanca (eurocéntrica), igualitaria y excepcional de la nueva y permanente patria. La siguiente sección examina tales relaciones entre un proyecto de concientización étnica transnacional, nacional costarricense y cosmopolita en el discurso de Alex Curling Delisser, hijo e intelectual de su contexto y comunidad.

CURLING, INTELECTUALIDAD Y CONCIENTIZACIÓN A TRAVÉS DE LA PRENSA

Nacido en San José, el primer diputado afrocostarricense, el abogado Alexander Curling Delisser, se naturalizó el 11 de septiembre de 1935. En el acta hizo constar que era casado, en aquel entonces estudiante -de derecho-, de progenitores jamaiquinos, vecino de San José y nacido allí el 3 de septiembre de 1908. Sin embargo, mucho antes de asumir su cargo político, Curling atravesó serias dificultades para defender los derechos individuales y colectivos de la población de descendientes de migrantes caribeños. Prueba de ello fue lo concerniente a permitir la libertad de desplazamiento de dicha población hacia el Valle Central (Senior 154).

El 10 de marzo de 1936, dos años después de padecer la prohibición para sumarse a la fuerza laboral de las compañías bananeras en el Pacífico, por razón del color, y a tan solo seis meses de haberse convertido en ciudadano, Alex Curling Delisser publica el artículo "Todo hombre 
es igual ante la ley" en el diario La Tribuna. El texto evidencia cómo las dinámicas de racismo en Costa Rica no solo condicionaban las oportunidades de circulación y movilización de pobladores negros hacia el Pacífico, sino incluso su presencia dentro de la provincia en donde se "aceptaba" su reclusión. Específicamente, Curling cuestiona un oficio de la Municipalidad de Limón "que prohíbe el uso del Balneario Municipal a un buen número de ciudadanos costarricenses por el solo hecho de pertenecer a la 'raza' de color" (Curling, "Todo hombre" 14). En el caso centroamericano, las experiencias explícitas de segregación son registradas por la historiografía para el caso de Panamá y su división racial (de plata y oro) en el Canal (Bourgois 136-137). Por su parte, la segregación que la población costarricense esbozaba en su imaginario se traduce tanto en la resolución municipal y conjunto de legislaciones restrictivas, como en una serie de leyes "fantasmas", acuerdos tácitos y racistas, sobre los límites de la circulación negra y el uso de espacios públicos y recreativos ${ }^{12}$.

Alex Curling, "ciudadano costarricense por un acto de voluntad", reclama entre sus obligaciones el elevar su "voz de protesta contra una medida que lesiona los derechos inviolables de mis [sus] conciudadanos" (Curling, "Todo hombre" 14). Su apología integra las premisas de la democracia costarricense y del mismo nacionalismo hegemónico; por ello, exalta las virtudes de una patria que siendo coherente con sus valores de justicia deberá desterrar las manifestaciones del racismo -que se acusa como anomalía- y reconocer el derecho de quienes, nacidos en sus tierras, no gozan aún de la condición plena de ciudadanía. La lucha por la igualdad de los ahora "costarricenses de color", a quienes Curling representa desde una intelectualidad negra-caribeña, constituye, a su vez, una apuesta de confianza en la institucionalidad democrática. Dado que la intelectualidad étnica es consecuencia tanto de la contingencia

12 Como lo ha recuperado Soto Quirós, los ejemplos de legislaciones racistas incluyen las restricciones migratorias de 1940 que hacen una equivalencia entre nacionalidades, condiciones de "raza" y estado mental y de salud para definir, según el Registro Civil, las "prohibiciones de ingreso o transeúntes de 'raza' negra, chinos, árabes, sirios, armenios, gitanos, coolíes, etc [...] que padezcan de enfermedades mentales, venéreas, tuberculosis [...] tahures, vagos, rateros [...] mendigos, valetudinarios [...] toxicómanos [...] personas inconvenientes, nocivas o peligrosas al orden o progreso de la República o a la conservación de la 'raza', ya sea por sus tendencias agitadoras... o por las características que predominen en ellas y sean de notoria desafinidad con la población nacional” (cit. en Soto Quirós 128). 
material en que está situada, como de las estructuras de poder presentes en la sociedad mayor de la que es resultado (González 294), el abogado demanda la coherencia entre la gestión estatal y los mismos valores fundacionales de la patria pregonados por la narrativa nacionalista; además, encomia su plausible ejercicio.

Su argumento como ciudadano de color, abogado y vocero de la igualdad y la justicia, cobra particular relevancia en la antesala de la década de los cuarenta, quizás el periodo de mayor agitación en la vida política nacional costarricense del siglo XX. Se trata de la era de la conquista de las llamadas "garantías sociales" que facilitan el acceso a la educación, salud y consecuente ascenso social de una clase trabajadora y campesina que llegará a incluir a pobladores negros caribeńos cuya educación y profesionalización continúa en la capital de San José. Al filo de esta década, y en consonancia con las dinámicas geopolíticas, el país sufre también una fractura, más partidista que ideológica, que desencadenará en la última guerra civil de Costa Rica y el inicio de la Segunda República. Para Senior, la "democrática naturaleza" de Costa Rica se enfatiza después de la Guerra Civil de 1948, cuando el grupo hegemónico celebra la fundación de una verdadera "democracia social" en donde se vislumbra un lugar "para la población afrocaribeña del país... beneficiarios de las nuevas 'garantías' concedidas" (197).

Curling critica las manifestaciones de racismo a nivel local, en conexión con dinámicas internacionales de supremacía blanca de larga data y exacerbadas en la antesala de la Segunda Guerra Mundial. También, señala las inconsistencias entre tales expresiones y la trayectoria de la pequeña democracia, cuya tradición igualitaria se celebra "pues ofrecemos a los ojos del mundo el ejemplo de un país que ha sabido encauzar su vida por los senderos de la libertad, la igualdad y la fraternidad, y en donde la paz, que en otros países no pasa de ser una aspiración, es en el nuestro una hermosa realidad" (Curling, "Todo hombre" 16). Quizás en un afán persuasivo y laudatorio de las cualidades -ilustradas- costarricenses, Alex Curling sugiere que, como amenaza foránea "ahora es el prejuicio racial con su odio de 'razas' el que pretende manchar nuestro cielo..." (14) e invita a la razón ilustrada a prevalecer. Más allá de las posibles inconsistencias (colonialistas) en su discurso ${ }^{13}$, el texto permite inferir

13 En esta dirección, Rosario analiza el discurso inaugural de Alex Curling en la Asamblea Legislativa como resultado de una identidad, visión de grupo 
ciertas características de un estilo de incidencia política que articula su concientización étnica transnacional, nacional costarricense e incluso cosmopolita. En esa dirección, es relevante cómo Curling alude a los ideales de la Revolución Francesa, como veremos adelante, pero llama la atención en su texto el manejo simultáneo de referencias supranacionales de lucha antirracista mediante la evocación de cierto "panteón negro". Su listado incluye no solo figuras políticas, sino otros nombres sobresalientes en el ámbito cultural panafricano, entre ellos: "Hombres de letras como Juan Latino, el célebre poeta español; Alejandro Dumas; Paul Lawrence Dunbar y James Weldon Johnson; educacionistas como Brooker T. Washington; libertadores como Antonio Maceo y Touissant Louverture; cantantes como Paul Robson y Roland Hayes; compositores como Samuel Taylor Coleridge y atletas como Jesse Owens y Joe Louis" (14). Como evidencia de una erudición política y negra, el autor repetirá tal recurso de evocación de figuras célebres a lo largo de la diáspora en su discurso de juramentación como diputado en 1953. Volviendo al gesto de negociación transnacional-nacional, Curling asume la consigna de ennegrecer al héroe nacional, Juan Santamaría, junto a las referencias internacionales ${ }^{14}$. Reconoce, en su conjunto, "a la 'raza' que ha dado en América héroes como Touissant Louverture, Antonio Maceo y Juan Santamaría, a escritores como Alejandro Dumas, a artistas como Marian Anderson" (cit. en Rosario 251). Es decir, reduce las figuras rememoradas, aunque incluye esta vez a una mujer y a quien, desde su heroicidad y presencia, pondría en entredicho los mismos discursos de la nación blanca: Santamaría, el tamborcillo de Alajuela.

A título personal, entre 1949 y 1950 Curling se dio a la tarea de denunciar los impedimentos que pesaban, de manera particular, sobre la naturalización de la población de color, así como de concientizar políticamente, mediante la expresión manifiesta y articulada de su

y de la sociedad en su conjunto. Para ella "de todo discurso se derivan representaciones culturales, estereotipos y otras imágenes sociales sobre ciudadanía", que en el caso de Curling incluye la necesidad de demostrar su condición costarricense (251-253).

14 Sobre los procesos selectivos en la construcción del héroe Juan Santamaría, ver Ríos: "De conflictos y ambigüedades: estrategias de representación del héroe costarricense Juan Santamaría". 
pensamiento ${ }^{15}$. Curling utiliza el espacio periodístico como plataforma de denuncia antirracista y de conciencia negra, en continuidad con los circuitos del internacionalismo negro en donde la prensa cumple una función central ${ }^{16}$. Junto a la nota de La Tribuna, en 1936, la lista de publicaciones en prensa nacional incluye: "Los costarricenses de 'raza' de color" (La Nación, 20 de abril de 1949), "Que los negros de Costa Rica tengamos en mayor grado patria, cultura, justicia y libertad" (Diario de Costa Rica, 26 de junio de 1949), "Cives romanus sum" (La Nación, 6 de octubre de 1949), "Júbilo de los costarricenses de color" (La Nación, 9 de noviembre de 1949), "Apélase ante el Presidente de la República contra impuesto a súbditos británicos de color" (Diario de Costa Rica, 27 de agosto de 1950), "Integración de las gentes de color a la nacionalidad y cultura costarricenses" (La Nación, 27 de octubre de 1950). Además, se cuenta con el artículo "Un triunfo para la democracia Costarricense" en The Atlantic el 19 de noviembre de 1949, publicado originalmente en el Diario de Costa Rica ${ }^{17}$.

La construcción de sus títulos enfatiza la viabilidad de una Costa Rica que abrace la ciudadanía negra, por ejemplo, en la medida que apela a la dimensión étnica y su condición de "raza", junto al estatus costarricense $\mathrm{e}^{18}$. Mediante la denuncia de las prácticas discriminatorias imperantes en la Costa Rica de mediados del siglo XX -en concreto, el sesgo racista a nivel de la institucionalidad responsable de los trámites migratorios-, Curling proyecta en un día (lejano) el destierro de la injusticia social, según se lee en el siguiente fragmento:

15 Dicha serie de artículos dieron fruto propiciando, entre otras cosas, la abolición "simbólica" de la restricción geográfica y concretando la apertura del proceso civil tendiente a la ciudadanía (Senior 155).

16 Para Putnam, la prensa en el circum-Caribe junto con las organizaciones cívicas negras crean una esfera pública, "a set of a physical or mediated spaces where people can gather and share information, debate opinions, and tease out their political interests and social needs with other participants (Radical 147).

17 Los archivos de prensa originales fueron consultados y están disponibles en la hemeroteca de la Biblioteca Nacional. Para este artículo, las citas seleccionadas corresponden a la compilación de Curling Rodríguez en el texto Alex Curling Delisser, Benemérito de la Patria: Padre de la igualdad jurídica.

18 Para una mayor comprensión de las particularidades de la noción de ciudadanía negociada por Curling y su comunidad, se recomienda la consulta del libro de Senior: Ciudadania Afrocostarricense: el gran escenario comprendido entre 1927 y 1963. 
Comprobada la veracidad del cargo que formulé, y a fin de que renazca la confianza del elemento de color en la imparcialidad y corrección de los funcionarios públicos, estimo que no sería mucho pedir [...] una declaración pública en el sentido de que el Ministerio de Relaciones Exteriores de la actual administración recibirá y dará curso a las solicitudes de naturalización que presenten los habitantes de color de acuerdo con la ley [...] en no lejano día será desterrada de la administración pública la injusticia social de la discriminación vigente (Curling, "Los costarricenses" 19).

Curling articula su discurso en términos de verdad, rendición de cuentas y lucha contra la injusticia social. Con ello, no solo evidencia su profesión, sino la relación especial entre el intelectual crítico y comprometido y la historia y política de su tiempo, una función que va más allá del individuo y su formación (Said 20). Ahora bien, en la medida que su lucha trasciende el contexto local y consciente de un sistema racista que opera a nivel supranacional, Curling expande sus estrategias persuasivas. Puede distanciar la experiencia del pequeño país de otros contextos de mayor violencia racista o bien, como otros intelectuales de su época, apelar a un discurso de libertad panamericanista y abolicionista para "que los negros de Costa Rica tengamos en mayor grado, cultura, justicia y libertad", tal cual escribe dos meses después en el Diario de Costa Rica y que recuperamos en el siguiente fragmento:

Y que lleve así su cabal realización en nuestra Patria la obra redentora de Lincoln y Bolívar. [...] La abolición de la trata de negros y la esclavitud, máxima negación de los derechos humanos[,] es la primera etapa de un largo proceso de liberación que aún no ha terminado: a los negros todavía se nos humilla y maltrata con grilletes como el que nos colocó un Congreso de la República a los ciento diez ańos de haber abolido Centroamérica el régimen oprobioso e inhumano de la esclavitud. Vengo por este medio a pedir la protección de la Liga para la Defensa de los Derechos del Hombre, y no solo de esta humanitaria organización, sino también la de todos los hombres de buena voluntad, para que los negros de Costa Rica tengamos en mayor grado, cultura, justicia y libertad, y se lleve así a su cabal realización en nuestra patria la obra redentora de Lincoln y Simón Bolívar (Curling, "Que los negros" 21). 
Sin dejar de apelar al sentimiento patrio, y desde su identidad negra, Curling convoca a las figuras de Abraham Lincoln y Simón Bolívar. Si anteriormente acudía a personajes de la diáspora para afirmar la condición honorable de su raza, la cruzada antirracista requiere, también, la apelación (soteriológica) del abolicionista del Norte. A su vez, y desde una mirada hemisférica que confirma la circulación de las ideas sobre raza en el continente (Hooker 2), el binomio de líderes se completa con la mención del libertador de América, lo cual sugiere un conocimiento erudito de Curling acerca de la figura de Bolívar y su Carta de Jamaica. Tales referencias enmarcan las nociones de justicia y libertad del autor y, en última instancia, procuran darle peso al reclamo de derechos humanos ante la Liga para la Defensa de los Derechos del Hombre por causa de "la ignominiosa ley $\mathrm{N}^{\circ} 30$ del 10 de diciembre de 1934 la prohibición de emplear gentes de color en la industria bananera del Pacífico" (Curling, "Que los negros" 21).

Ahora bien, retomando las ideas de la llamada intelligentsia étnica y el ejercicio profesional de la inteligencia ${ }^{19}$, Curling "producto tanto de la epistemología en la que fund[a] su comportamiento, como por los grados y tipos de instrucción adquirida, pued[e] presentar comportamientos [...] etnicistas, propios de la intelligentsia, como también pued[e] mostrar conductas [...] cosmopolitas, propi[a]s de la intelectualidad" (González 293). La misma versatilidad en el manejo de fuentes discursivas y referentes políticos y culturales sintetiza esa confluencia. Para comprender el alcance de un conocimiento "cosmopolita" de la mano de una perspectiva de la longue durée, la siguiente nota publicada por Alex Curling en La Nación, el 6 de octubre de 1949, merece particular atención.

Cives romanus sum (Soy ciudadano romano). Fue esa frase el escudo que opuso San Pablo a sus perseguidores y la llave de oro que abrió al Santo las puertas de la justicia romana. Simboliza esa histórica frase de los romanos la más hermosa y elevada expresión del genio político de Roma, que más que un poderoso imperio, creó y enalteció como ningún otro pueblo de la Antigüedad el concepto sublime de ciudadanía. [...] Siglos más tarde volvió esa frase con dramático efecto en el seno del

19 Gramsci vinculó inicialmente el concepto "intelectual" a la capacidad racional de los seres humanos, ya que, según él, todos somos intelectuales por la capacidad humana de pensar; sin embargo, solo algunos se dedican al ejercicio profesional de esta actividad. 
Parlamento Inglés. Corría el ańo de 1850. El gobierno de Lord Rusell era duramente criticado por la forma enérgica con que había procedido en el incidente internacional a que había dado lugar el ultraje sufrido por un judío de nacionalidad británica residente en Grecia. Hizo la defensa del gobierno el Ministro de Relaciones Exteriores, Lord Palmerston, en un discurso que es considerado como una obra maestra de polémica parlamentaria. Defendió brillantemente el derecho de los Estados de proteger a sus súbditos de tierras extrańas [...] Es Francia, indiscutiblemente, la nación que en los tiempos modernos ha devuelto al concepto de ciudadanía su antiguo contenido, iluminándolo con los fulgores de una Revolución que proclamó los principios de la igualdad, libertad, fraternidad, y consecuente con esos postulados, abolió la esclavitud y declaró que "todos los hombres, sin distinción de color, domiciliados en las colonias, son ciudadanos franceses y gozarán sus derechos". [...] El concepto de ciudadanía, tal como lo entendieron los romanos y lo aprecian los franceses, fue interpretado recientemente por el Presidente Truman cuando dijo que la calidad de un buen ciudadano no se juzga por la cuna, la estirpe, la religión ni la "raza", sino por el carácter, la cultura, la cooperación y la lealtad. [...] defensor de los derechos cívicos del negro norteamericano, al extremo de anteponer tan laudable labor a los legítimos intereses políticos. [...] Como Francia y los Estados Unidos, Costa Rica es una república fundada en los principios que consagró la Revolución Francesa, y por consiguiente dedicada a la proposición de que todos los hombres nacen iguales. Más de cien ańos de vida republicana y una historia iluminada por los resplandores de la tea que en sus manos sostuviera un humilde y moreno tamborcillo de Alajuela, ha[n] imprimido a la ciudadanía costarricense un prestigio que no cede en grado al de ningún otro pueblo antiguo o moderno de la Tierra. [...] "con el apodo de El Erizo. Aquel negrito hizo una hazaña que los ticos blancos difícilmente podrían igualar, que no superar, en lo futuro. Santamaría es una gloria nuestra. Es el Maceo costarricense". [...] La primera batalla de esta cruzada ha sido librada y ganada en la Asamblea Constituyente, cuyos miembros han aprobado ser dignos baluartes del civismo nacional. [...] Pero, aunque sucediera lo inconcebible, aunque los negros, cual esclavos de antaño, tuviésemos que seguir por más tiempo marcados con el hierro candente de una discriminación odiosa, el ciudadano de color, puesta su mirada en la Patria que adora, en la tierra gentil y madre de amor que exalta el Himno 
Nacional, siempre dirá: "Soy ciudadano costarricense" con la misma fe y con el mismo orgullo con que el ciudadano romano exclamaba: Cives romanus sum (Curling, "Cives" 23-26).

Pese a una apariencia sencilla y accesible en su secuencia narrativa, el texto de Curling denota una alta complejidad y sofisticación en cuanto al manejo de datos históricos y el encadenamiento de figuras y sucesos. En primer lugar, la exposición parte del intertexto bíblico, el cual, a su vez, sugiere la comprensión de la Europa moderna como continuación del llamado mundo clásico (Dussel 44). Luego, la evocación del discurso de Lord Palmerston ante el Parlamento Inglés en 1850, cuya referencia la misma Putnam identifica como recurso retórico dentro de los reclamos de vinculación imperial de ciudadanos británicos negros a lo largo del circum-Caribe en el periodo entre guerras (Radical 41). La imagen sugiere un tejido de relaciones intertextuales-políticas que se actualiza en contextos de abandono y desprotección ciudadana, tal cual corresponde a la precaria oportunidad de nacionalización de los costarricenses de color al filo de la década de los cuarenta. Ahora bien, la apelación a los valores de la modernidad eurocéntrica continúa en su escrito y Curling establece la conexión entre el concepto de ciudadanía romana, los valores ilustrados de la Revolución Francesa y sus principios universales abrazados en el nuevo mundo, tal cual lo confirma la cita del presidente Truman. La adopción de un conocimiento "cosmopolita", en relación con el continuum de la modernidad instaurada como verdad universal, no parece contradictoria considerando el contexto y la instrucción recibida por el intelectual. Sin embargo, llama la atención el mecanismo por el cual, mediante un aparente recorrido lógico y cronológico, los mismos valores ilustrados que se inspiran en la Antigüedad clásica se adoptan de igual forma desde el Norte hasta el Centro de las Américas. Curling pareciera estar haciendo un guińo a los imaginarios democráticos (y de excepcionalidad) costarricense al hacer un parangón entre Roma, Francia, Estados Unidos y Costa Rica.

Seguidamente, el autor anticipa la estrategia de apropiación del héroe de tez oscura mencionada arriba -Juan Santamaría- para apelar a un nacionalismo en donde la ciudadanía negra no es solo posible, sino deseable. Luego de una secuencia discursiva eurocéntrica y, por ende, blanca, valida los valores del "humilde y moreno tamborcillo de Alajuela", "con el apodo de El Erizo", junto al mismo brillo y prestigio de "cien años de vida repu- 
blicana" y de la "ciudadanía costarricense". El hito de su apelación, racional, pasa por el combate del prejuicio histórico y la afirmación de un legado de los hijos de la diáspora para la consolidación de las naciones americanas y caribeñas. Se comprende entonces por qué, como se mencionó anteriormente, al llegar a la Asamblea Legislativa el panteón negro evocado por Curling incluye, junto a Toussaint Louverture y a Antonio Maceo, a un ciudadano costarricense negro y heroico: "Santamaría es una gloria nuestra. Es el Maceo costarricense".

Desde el conocimiento universal, europeo, americano, regional circum-caribeño, local, pero ante todo, étnico, el intelectual esboza una justificación infranqueable para garantizar los derechos de su comunidad. Así lo demuestra a lo largo de varios ejemplos en su escritura periodística. Curling apela a referentes de la historia universal (cosmopolita), continental -como lo hace con la imagen de Simón Bolívar-, pero también nacional; ya sea mediante la mención al héroe negro o bien a otras figuras clave dentro de las fuerzas políticas en pugna y con quienes "simpatizará" políticamente. Así, un mes después de la escritura del Cives romanus sum, el 9 de noviembre de 1949 Curling relatará, en cierto tono poético, en $\mathrm{La}$ Nación, la celebración de:

la derogatoria de la discriminación legal que coartaba nuestra libertad de trabajo en la zona del Pacífico. [...] por su trascendencia en el orden moral, ya que la patriótica acogida que la Junta Fundadora de la Segunda República ha dispensado al gesto sublime de la Asamblea Nacional Constituyente reafirma nuestra fe en el espíritu cristiano y democrático del pueblo costarricense; da contenido real a la brillante defensa que de los derechos del hombre hiciera Costa Rica en la Organización de las Naciones Unidas; constituye un motivo más de profundo agradecimiento para quienes expusieron sus vidas y haciendas por devolverle a la Patria sus más preciadas libertades; y sobre todo, dice al costarricense de color, con la sinceridad sublime de los hechos, que la ciudadanía que ostenta es en verdad el galardón de que hablara Omar Dengo en su vibrante defensa de la "raza" de color, la llave de oro que abriera a San Pablo las puertas de la justicia romana, el título que glorifica Simón Bolívar ante los pueblos de América, y el tesoro inapreciable por el que derramaron su sangre sacrosanta los héroes de Santa Rosa y Rivas (Curling, "Júbilo" 27). 
El abogado manifiesta su regocijo por la eliminación de una ley que resulta central desde los inicios de su intelectualidad y accionar, tal cual deja manifiesto en su artículo "Que los negros de Costa Rica tengamos en mayor grado patria, cultura, justicia y libertad" ya referido. En esta nueva intervención en prensa, y desde un tono celebratorio, desea encontrar eco en figuras nacionales de renombre como Omar Dengo y los héroes de Santa Rosa y Rivas (entre ellos, Juan Santamaría); así como en la institucionalidad nacional de la Junta Fundadora de la Segunda República y la Asamblea Nacional Constituyente. Estas referencias resultan cruciales considerando que, el día anterior a la publicación del artículo, se promulga la nueva Constitución Política (7 de noviembre de 1949). Ahora bien, junto a esta maniobra de situar su discurso en el plano nacional, vuelve a convocar a nombres de referencia hemisférica. Rescata una vez más la figura de San Pablo y Simón Bolívar e, inclusive, la incipiente institucionalidad de la Organización de las Naciones Unidas.

El proceso de reivindicación de una comunidad negra en Costa Rica es gradualmente asegurado desde sus textos. Casi un año después, pensándose él y su comunidad como parte de la nacionalidad costarricense, confirma lo siguiente en su texto igualmente publicado en La Nación, bajo el título "Integración de las gentes de color a la nacionalidad y cultura costarricenses":

El negro es piedra de toque de nuestra democracia; porque democracia que lo oprima no es la democracia cristiana que vivieron tan dignamente el presbítero Florencio del Castillo, el educador Omar Dengo y el Presidente Ricardo Jiménez. Ábranse de par en par las puertas de la nacionalidad y cultura costarricenses a las gentes de color y[,] como los vislumbra el visionario de Omar Dengo, Costa Rica tendrá en la zona del Atlántico "un tesoro moral de[l] que ahora carecemos y un tesoro material también" (Curling, "Integración” 34-35).

El artículo aparece cuando aún distan tres años de la llegada de Curling al Congreso, pero sugiere ya un acercamiento al terreno político estatal, evidente desde la repetida mención a Omar Dengo y al Presidente Jiménez. En este proceso de alianzas políticas, el abogado goza además del respaldo de la población negra, como consta en una "carta abierta" publicada en El Correo del Atlántico, el 28 de mayo de 
1949, cuatro años antes de ser Diputado y justo en el año en que se amplían las categorías de ciudadanía en el país. Miembros de la comunidad e intelligentsia afrocaribeña, quienes participan de los espacios de activismo y circulación de las ideas políticas negras, como la UNIA y los periódicos locales, le manifiestan su simpatía y agradecimiento:

Carta Abierta al Lic. don Alex Curling

Limón, CR, abril 22, 1949

Lic. Alex Curling

San José

Muy Estimado Amigo:

Nosotros los suscritos deseamos por este medio congratularlo muy calurosamente por la valiente lucha que Ud. está conduciendo contra la discriminación y en la defensa de los derechos de la Raza de Color.

Deseamos de todo corazón que le sea prestada la vida para continuar esta digna obra humanitaria empezando por Ud.

Que Dios lo bendiga.

Orlando Williams, Edwin Jackson B., Alfonso Taylor, Clarence J. Sankey, Alex Campbell, Arturo Corvand, E. Barley, Johnny Steele, Consuelo Campbell, Chas Edwards, K. J. Moore C., Peter A. Muir, Leslie H. Angus, Albert W. Splatt, Thos B. Lynch (Mayor), Stan E. Dixon D. (UNIA), P. Booth, J. P. Holmes, Jos A. Thomas (editor del periódico The Atlantic/El Atlántico) (Senior 262, el énfasis es nuestro).

Una vez asegurada la ciudadanía de "los costarricenses de raza de color" por vía constitucional, líderes de la comunidad identifican la participación política como el paso necesario para el ejercicio pleno de derechos y deberes. Además, el ingreso a la institucionalidad aspira a la inclusión de una agenda que favorezca a estas poblaciones de color, de manera particular, en la provincia de Limón. Sobre las vías de participación, la incorporación a alguno de los partidos políticos tradicionales fue considerada como la ruta más viable y efectiva (aunque no la única) para la incidencia; concretamente, con perspectivas de llegada al primer poder de la República, la Asamblea Legislativa de Costa Rica. En esa dirección, aparece el llamado "Black Whiz", un grupo de negros con aspiraciones políticas, entre quienes destacan la misma figura de Alex 
Curling Delisser y Stanley Britton. Sawyers explica su gestión para procurar un espacio -que quizás no significará una cuota de poder-en la contienda de 1953.

Antes de 1948, acaso votaban 30 negros. En 1951, con la cercanía de las elecciones de 1953, un grupo de negros que se llamaron a sí mismos "Black Whiz", que significa "negros sobresalientes", escribieron una carta a los tres partidos políticos sobresalientes, Unión Nacional, Republicano Nacional y Liberación Nacional, diciendo que ellos sentían que era esencial que los negros que ahora eran costarricenses debían y tenían el derecho de participar en la política del país.

Del Partido Unión Nacional recibieron un "No" rotundo. No querían tener nombres ni apellidos extranjeros en sus papeletas porque los dañaría. Del Republicano recibieron la misma negativa. Del Partido Liberación Nacional recibieron en cambio un cálido sí. "Ustedes son ahora ciudadanos costarricenses y deben ser incluidos en cualquier lugar y posición de la papeleta". Bienvenidos. De esta forma el negro empezó a participar en la política costarricense, con su iniciativa (cit. en Meléndez y Duncan 230-231, el énfasis es nuestro).

En el fragmento anterior, incluido en uno de los primeros estudios sobre la comunidad de descendientes jamaiquinos y la presencia negra en territorio nacional, K. Sawyers ${ }^{20}$ rescata la agencia del grupo "por su iniciativa" en reclamar un espacio en las esferas de decisión política que conllevará a la elección del Diputado (suplente) Alex Curling en 1953. Gracias a esta gestión, en palabras de Duncan y Allen, "desde la fundación de la Segunda República, siempre hubo una Curul, ocupada por un diputado o diputada de la comunidad negra en la Asamblea Legislativa en representación de la provincia de Limón por el Partido Liberación Nacional" (92) 2 .

Hermana de quien llegará a ser diputada para el periodo 1998-2002, Joycelyn Sawyers Royal, y que fue mencionada en la introducción de este artículo.

21 La alianza es relevante cuando se considera que este partido mantiene una trayectoria de dominancia en el panorama electoral del país. Sin embargo, Rosario es crítica sobre la respuesta del mismo PLN y de la negativa del resto de partidos. En su análisis de la negociación, la misma negativa del resto de los partidos da cuenta de la resistencia general a la participación negra en los espacios de poder estructurados desde el nacionalismo blanco costarricense. Desde su análisis, el 
El caso de Curling como intelectual de una comunidad que -al igual que otras de la "raza" de color- enfrenta la resistencia al reconocimiento de sus derechos de ser y ejercer ciudadanía, sugiere que el ingreso a la política no es incompatible con su conciencia étnica. Su presencia en el espacio de la representación política no supone tampoco el destierro de los imaginarios de la nación blanca. No obstante, aun desde su condición marginal en el Congreso, Alex Curling Delisser ejerce una ardua lucha por la concreción del derecho de ciudadanía para los descendientes de afrocaribeños y demás minorías étnicas que conforman la nación costarricense, y por el mejoramiento de las condiciones de desarrollo de la provincia de Limón (Rosario 254-255).

El paso de la tribuna de la prensa a la esfera legislativa es coherente en términos de su discurso político de patria, justicia y libertad. Específicamente, la llamada "Ley Curling" contribuyó con la reforma a las leyes de Migración y Extranjería y permitió a los ciudadanos extranjeros, que vivían en el territorio, optar por la nacionalidad costarricense. En palabras de la proponente del proyecto de su benemeritazgo, la exdiputada Joycelyn Sawyers -y como consta en el Acuerdo 6041-2001 de la Asamblea Legislativa de Costa Rica-, Curling "se esforzó por la igualdad jurídica de los ciudadanos extranjeros, que constituían minorías en este país [...] Fue un defensor de la Declaración Universal de los Derechos Humanos y de sus principios fundamentales: libertad, justicia y paz" (Asamblea Legislativa 54). La gestión de Alex Curling Delisser en el Congreso incluye además el proyecto de construcción de la carretera hacia Guápiles, que enlaza el Caribe a la capital, San José y otras obras de infraestructura en beneficio de la provincia de Limón. Se trata de iniciativas que reclaman y transforman la experiencia de las poblaciones negras en la nación costarricense. Ahora bien, mientras sus contribuciones en el espacio de la legislación migratoria y la inversión social

PLN tampoco se libra de dichas actitudes racistas, sobre todo si se considera el proceso mediante el cual Alex Curling participa activamente en la campaña presidencial de José Figueres Ferrer en 1953 y, aunque merecedor de encabezar la papeleta de diputación, es nombrado suplente. El diputado titular William Reuben se retira de la sesión del 2 de noviembre de 1953 y permite el ejercicio de Curling durante ese día y durante la mayoría de su periodo. Será hasta casi una década después de la participación de Curling que se elija un diputado negro como titular (248-255). Estas consideraciones dan cuenta de las complejidades del escenario de participación política de la población negra. 
han sido reconocidas por la comunidad afrocostarricense - con grandes desafíos mediante el benemeritazgo-, el conocimiento de su propuesta intelectual se encuentra en proceso germinal. La identificación de una confluencia entre referentes étnicos y locales, pero también cosmopolitas, en sus escritos y discursos se ofrece como una posible ruta de análisis.

\section{Conclusiones}

A lo largo de este artículo, se ha señalado cómo en un escenario más amplio de restricciones migratorias, ciudadanas y racistas de carácter circum-caribeńo, el pensamiento político de Curling sintetiza la interrelación entre el transnacionalismo negro, los procesos de negociación dispares entre la nación imaginada blanca y sus "ciudadanos de color" y la influencia de ideas ilustradas y del discurso moderno. Quien llegara a ser el primer diputado descendiente de antillanos y afrocostarricenses, forma parte de una intelligentsia étnica, esto es, "sujetos dedicados al ejercicio profesional de la inteligencia", quienes "producto tanto de la epistemología en la que fundan su comportamiento, como por los grados y tipos de instrucción adquirida, pueden presentar comportamientos [...] etnicistas, propios de la intelligentsia, como también pueden mostrar conductas [...] cosmopolitas, propi[a]s de la intelectualidad" (González 293). Alex Curling sintetiza estratégicamente $-y$ no sin tensiones-ambas posturas en la generación de un reclamo de justicia social, gracias al cual algunos representantes de su comunidad lo llegan a reconocer como "el continuador en Costa Rica de la obra de Marcus Garvey y predecesor de Martin Luther King" (Sawyers, "En emotiva ceremonia" 165).

Los discursos de Curling y de los colectivos con los cuales este se asocia, evidencian la circulación de una retórica antirracista de carácter diaspórico y de reclamo por el reconocimiento -adquirido por derecho en virtud de generaciones de trabajadores(as) - del aporte de la comunidad de migrantes afrocaribeños y sus descendientes a Costa Rica. Dicha vinculación entre el Caribe costarricense y la lucha transnacional negra, además de este discurso de contribución a la patria que se reconoce propia y en sintonía con los valores ilustrados, emergen en la lectura de la producción en prensa de este intelectual. 
Por un lado, el análisis de la intervención de Alex Curling Delisser brinda elementos sobre la continuidad entre los primeros esfuerzos de movilización de la comunidad afrocaribeña, su carácter transnacional antirracista y las iniciativas grupales y personales de participación y derechos ciudadanos. Por otro, su retórica da cuenta de las estrategias de negociación política entre el poder estatal, su liderazgo e imaginario blancos con su(s) interlocutor(es) negro(s), quienes reclaman igualdad en el estatus de ciudadanos costarricenses, durante los cuarenta y principios de los cincuenta. El primer diputado y benemérito de la patria de color emerge así, como un intelectual afrocostarricense de tradición negro-afrocaribeña que fue capaz de articular sus ideas, informadas y expansivas, con la acción política. Más aún, la figura y logros de este personaje (y su comunidad) evidencian los desafíos que enfrenta la primera generación de actores políticos negros en su esfuerzo por asegurarse un espacio en los imaginarios de la "blanca" nación democrática.

Bibliografía

Asamblea Legislativa de la República de Costa Rica. Acuerdo 6041-2001 "Declárese Benemérito de la Patria al licenciado Alex Curilig Delisser". San José, 2001. Impreso.

Bourgois, Philippe. Banano, etnia y lucha social en Centroamérica. San José, DEI, 1994.

Braudel, Fernand. La larga duración en la historia y las ciencias sociales. Madrid, Alianza, 1979.

“Centro Cultural Afrocostarricense”. La Voz del Atlántico. Limón, 16 de mayo de 1942. Impreso.

Chomsкy, Aviva. West Indian Workers and the United Fruit Company in Costa Rica, 1870-1940. Baton Rouge. Louisiana State University Press, 1996.

Curling Delisser, Alex. "Todo hombre es igual ante la ley". Alex Curling Delisser, Benemérito de la Patria: Padre de la igualdad jurídica, Maud Curling-Rodríguez (comp.), San José, Curling/Editorama S. A., 2005, pp. 13-16 
"Los costarricenses de "raza" de color". Alex Curling Delisser, Benemérito de la Patria: Padre de la igualdad jurídica, Maud Curling-Rodríguez (comp.), San José, Curling/Editorama S. A., 2005, pp. 17-19.

" "Que los negros de Costa Rica tengamos en mayor grado patria, cultura, justicia y libertad". Alex Curling Delisser, Benemérito de la Patria: Padre de la igualdad jurídica, Maud Curling-Rodríguez (comp.), San José, Curling/Editorama S. A., 2005, pp. 20-22.

. "Cives romanus sum". Alex Curling Delisser, Benemérito de la Patria: Padre de la igualdad jurídica, Maud Curling-Rodríguez (comp.), San José, Curling/Editorama S. A., 2005, pp. 23-26.

. "Júbilo de los costarricenses de color". Alex Curling Delisser, Benemérito de la Patria: Padre de la igualdad jurídica, Maud Curling-Rodríguez (comp.), San José, Curling/Editorama S. A., 2005, pp. 27-28.

"Integración de las gentes de color a la nacionalidad y cultura costarricenses". Alex Curling Delisser, Benemérito de la Patria: Padre de la igualdad jurídica, Maud Curling-Rodríguez (comp.), San José, Curling/Editorama S. A., 2005, pp. 32-35.

Duncan, Quince y Carlos Meléndez. El negro en Costa Rica. San José, Editorial Costa Rica, 2012.

Dussel, Enrique. "Europa, modernidad y eurocentrismo". La colonialidad del saber: eurocentrismo y ciencias sociales, Edgardo Lander (ed.), Buenos Aires, CLACSO, 2000, pp. 41-53.

González G., Jaime A. "Intelectualidad étnica. Propuesta teórico-metodológica de un objeto de indagación”. Revista Temas Sociológicos, $\mathrm{N}^{\circ} 23,2018$, pp. 273-305.

Gramsci, Antonio. La formación de los intelectuales, Ciudad de México, Grijalbo, 1967.

Hooker, Juliet. Theorizing Race in the Americas: Douglass, Sarmiento, Du Bois, and Vasconcelos. Oxford, Oxford University Press, 2017.

Hutchinson Miller, Carmen. The Province and Port of Limon: Metaphors for Afro-Costa Rican Black Identity. Heredia, EUNA, 2015.

IsAjIw, WsEvolod W. "Ethnic-Identity Retention". Ethnic Identity and Equality: Varieties of Experience in a Canadian City, Raymond Breton et al. (ed.), Toronto, University of Toronto Press, 1990, pp. 35-38. 
James, Winston. Holding Aloft the Banner of Ethiopia: Caribbean Radicalism in Early Twentieth-century America. Londres, Verso, 1998.

Molina Jiménez, IvÁn. Costarricense por dicha: identidad nacional y cambio cultural en Costa Rica durante los Siglos XIX y XX. San José, EUCR, 2002.

Muñoz Muñoz, Marianela. "Bilingüismo político: Afrocaribeñas en el estado blanco y multicultural costarricense (1978-2017)". Disertación doctoral, Universidad de Austin, Texas, 2018.

. "Afrocentroamericaneidades: dislocación del istmo y translocación caribeña y diaspórica”, Revista Estudios N³8, 2019, pp. 51-84. Web. Disponible en: https://revistas.ucr.ac.cr/index.php/ estudios/article/view/37400

Palmer, Steven. “Hacia la 'auto-inmigración'. El nacionalismo oficial en Costa Rica 1870-1930". Identidades nacionales y estado moderno en Centroamérica, Jean Piel (ed.), San José, Editorial de la Universidad de Costa Rica, 1995, pp. 75-85.

Premdas, Ralph R. Ethnicity and Identity in the Caribbean: Decentering a Myth. Notre Dame,

Indiana, Helen Kellogg Institute for International Studies, 1996.

Putnam, Lara. "Ideología racial, práctica social y Estado Liberal en Costa Rica”. Revista de Historia, Universidad de Costa Rica, №39, 1999, pp. 139-186.

. Radical Moves. Caribbean Migrants and the Politics of Race in the Jazz Age. Chapell

Hill, University of North Carolina Press, 2013.

Quesada Soto, Álvaro. Uno y los otros. Identidad y literatura en Costa Rica 1890-1940. San José, Editorial de la Universidad de Costa Rica, 1998.

Ríos Quesada, Verónica. "De conflictos y ambigüedades: estrategias de representación del héroe costarricense Juan Santamaría”. Brújula. Revista Interdisciplinaria de Estudios Latinoamericanos, $\mathrm{N}^{\circ}$ 9, 2012, pp. 98-124.

Rosario Fernández, Reina Cristina. Las identidades de la población de origen jamaiquino en el Caribe costarricense en la $2^{a}$ mitad del siglo $X X$. Santo Domingo, Cocolo Editorial, 2015. 
SAID, Edward W. "The limits of the artistic imagination and the secular intellectual”. Macalester International, N³, 1996, pp. 3-34.

Sawyers Royal, Joyce. "Comunicación personal". 27 de septiembre de 2016.

."En emotiva ceremonia develan retrato de Alex Curling". Alex Curling Delisser, Benemérito de la Patria: Padre de la igualdad jurídica, Maud Curling-Rodríguez (comp.), San José, Curling/ Editorama S. A., 2005, p. 165.

Senior Angulo, Diana. Ciudadania Afrocostarricense: el gran escenario comprendido entre 1927 y 1963. San José, EUNED/EUCR, 2011.

Soto Quirós, Ronald. "Discursos y políticas de inmigración en Costa Rica. 1862-1943”. Iberoamericana, N 5, 2005, pp. 119-133.

Recepción: 28-09-20

Aceptación: 14-12-2020 